\title{
La Cultura Escolar: Concepto Clave para Entender la Implicación Escolar
}

\author{
School Culture: A Key Concept to Understand School Engagement
}

Jon Xabier Basogain-Urrutia ${ }^{1}$

\section{(2) EDICIÓN: \\ RTED \\ nsayos}

Recibido: 5/abril/2021

Aceptado: $10 /$ julio/2021

Publicado: 29/agosto/2021

\section{País}

${ }^{1}$ España

IIIIInstitución

${ }^{1}$ Toki Eder Ikastola

\section{$\lambda_{\varnothing}$ Correo Eletrónico}

1jonxabier.basogain@tokieder.eus

\section{(iD) ORCID}

${ }^{1}$ https://orcid.org/0000-0002-1454-6798

\section{Citar así: C APA / IEEE}

Basogain-Urrutia, J. (2021). La Cultura Escolar: Concepto Clave para Entender la Implicación Escolar. Revista TecnológicaEducativa Docentes 2.0, 10(2), 13-20. https://doi.org/10.37843/rted.v10i2.218

J. Basogain-Urrutia, "La Cultura Escolar: Concepto Clave para Entender la Implicación Escolar", RTED, vol. 10, n. ${ }^{\circ} 2$, pp. 13-20, ago. 2021

\section{Resumen}

La investigación educativa en los últimos años está prestando mayor atención a la implicación escolar. Se trata de una herramienta valiosa para conocer la relación de los estudiantes con la escuela, así como su grado de implicación con la misma. La implicación escolar, como herramienta de medición, aporta datos de gran valor para entender cómo se desarrolla esa implicación y qué variables interfieren en ella; además, ha quedado sobradamente demostrado que funciona como excelente indicador de problemas futuros como el abandono escolar. Aun así, tradicionalmente se ha ignorado el contexto escolar en el que se han recogido los datos. Se han tenido en cuenta algunos datos que atañen a las características del centro (nivel socioeconómico, lugar, edad del alumnado...), pero se ha obviado todo lo que sucedía dentro de la escuela. Así, este trabajo defiende que los datos de la implicación escolar deben estar sustentados por el conocimiento de la cultura escolar. De lo contrario, todo intento de entender o justificar la implicación, sin conocer la realidad o la cultura escolar, estará basado en teorías e hipótesis que se alejan del contexto real. En este ensayo se propuso un nuevo enfoque que destaca la necesidad de poner en valor la cultura escolar como concepto clave para entender la implicación en la escuela. Se diseñó un modelo novedoso de siete variables donde la implicación escolar y el ambiente de la escuela (la cultura escolar) son estudiadas conjuntamente, de tal manera que se consigan los datos necesarios para establecer relaciones causa-efecto y formular hipótesis adecuadas.

Palabras clave: Cultura escolar, clave, entender, implicación escolar.

\section{Abstract}

Educational research is paying greater attention to school engagement in recent years. It has become a valuable tool for analyzing the relationship of students with their schools, as well as their degree of engagement with them. School engagement, as a measurement tool, provides valuable data for the understanding of how this engagement develops and what variables affect it. In addition, it has been thoroughly confirmed as an excellent indicator of future complications, including school dropout. Despite these successes, an important element of the research, the school context in which the data was collected, has traditionally been ignored. Some data concerning the characteristics of the center (socioeconomic level, place, age of the students ...) have been considered. However, the events occurring within the walls of the school have been regularly ignored. Thus, this essay argues that the data on school engagement should be supported by knowledge of the school's culture. Otherwise, any attempt to understand or justify engagement, not knowing the concrete reality or the school's culture, will be based on theories and hypotheses that may be far from the real context. In this essay, a new approach of study is proposed. It highlights the need to value school culture as a key concept to understand school engagement. In addition, a novel model containing seven variables is presented, where school engagement and the school environment (school culture) are studied together. This model will provide the necessary data to establish cause-effect relationships and the formulation of adequate hypotheses.

Keywords: School culture, key, piece, school engagement. 


\section{Introducción}

Generalmente, cuando se habla de educación y de escuelas, se pone el foco en aquello que quizás resulte más fácilmente medible: los resultados académicos obtenidos por los alumnos, los informes del Programme for International Student Assessment (PISA), traducido al español como Programa Internacional para la Evaluación de Estudiantes $\mathrm{u}$ otros componentes o procesos de la educación alejados de lo que sucede dentro de la escuela. Son aspectos meramente académicos con características abstractas. Por ello, es indispensable también investigar otros conceptos inherentes de las escuelas.

En los últimos años, la comunidad educativa ha hecho un gran esfuerzo para entender tanto qué les pasa a los alumnos, como el modo de relacionarse con sus escuelas. Se ha tratado, incluso, de aunar aspectos académicos, emocionales y psicológicos investigando qué factores pueden propiciar el fracaso escolar. También se ha investigado la correlación entre la implicación escolar con otros factores como el cansancio escolar (conocido también como student burnout).

Uno de los conceptos más investigados, y que mayor relevancia ha tenido en los últimos años es, sin lugar a duda, el de la implicación escolar. Este concepto atiende a la manera que los alumnos tienen de relacionarse con su escuela e implicarse en la misma. Fredricks et. al. (2005) ofrece una definición del mismo. Esta es la más aceptada por la gran mayoría de autores donde se distinguen tres tipos de implicación:

1. Implicación emocional: caracterizada por recoger todas las reacciones emocionales, sean positivas o negativas, así como el sentimiento de pertenencia al centro. (Connell, 1990; Finn, 1989; Voelkl, 1997).

2. Implicación conductual: se centra en la participación del alumno en las actividades del centro; además se considera imprescindible para conseguir buenos resultados académicos (Connell, 1900; Finn 1989).

3. Implicación cognitiva: se refiere al esfuerzo que un alumno está dispuesto a llevar a cabo para entender tanto ideas como destrezas complejas. Hace referencia también al esfuerzo de ir un poco más allá del mínimo exigido (Corno \& Mandinach, 1983; Newman et. al. 1992).

Existen varios trabajos en este aspecto, tratando de ver qué factores pueden influir en la implicación escolar. Se ha comprobado, por ejemplo, que las variables edad o sexo influyen en el concepto de implicación escolar (Costa \& Tabernero, 2012; RamosDíaz et al., 2017). De igual modo, hay otras investigaciones sobre la relación de implicación escolar con otras variables como son el contexto familiar, el tipo de escolarización, y los problemas de conducta.

Sin lugar a duda, es un término de interés tanto para investigadores como para profesores. Ofrece una visión global de la relación de los estudiantes con su escuela, a la par que es un factor muy importante para predecir el abandono escolar (Connell, 1990; Finn, 1989). La manera de medir el nivel de implicación escolar actualmente puede llegar a ser escasa o, en cierto modo, imprecisa. Por ejemplo exponer, que "los alumnos de cierto barrio o centro tienen niveles de implicación cognitiva bajos", no aporta mucha información si no se investiga también el contexto escolar donde se han recogido esos datos. Se hace, por tanto, necesario investigar la cultura de esa escuela si se quiere explotar la información de los datos sobre implicación escolar; esto es, resulta imprescindible conocer qué sucede dentro de la escuela.

\section{Desarrollo}

Los trabajos de investigación sobre implicación escolar, en su gran mayoría, tienen en cuenta el contexto externo de la escuela recogiendo datos, entre otros, sobre nivel socioeconómico del lugar, ubicación, y origen del alumnado. Dicho proceso resulta lógico y se aplica en la mayoría de las 
investigaciones, sean del ámbito que sean. Además, es muy común que los datos se presenten atendiendo a tales criterios externos, como por ejemplo, las notas medias agrupadas por sexo, curso o la renta media del alumnado.

Esta realidad deja en evidencia que la investigación educativa no presta la suficiente atención a otros datos relacionados con lo que sucede dentro de la escuela, como qué valores, normas y costumbres rigen en el centro escolar, qué relación hay entre los alumnos, cómo de controlados están por la organización y cómo perciben los alumnos ese control. Los citados componentes de la vida escolar, que pueden influir en los niveles de implicación del alumnado, son muy relevantes y giran en torno a la vida dentro de la escuela. Pero "lo que sucede dentro de la escuela" no es un cúmulo de variables escogidas al azar, sino el resultado de todas las partes (costumbres, normas, suposiciones, creencias...) que conforman la cultura escolar.

Este concepto, el de cultura de la escuela, nace de aplicar un enfoque desde el punto de vista de la organización escolar a aquello que sucede dentro de la escuela. Sistematiza y categoriza aquellos fundamentos, procesos, factores $y$ componentes del día a día, que pueden pasar desapercibidos por ser parte de la rutina, pero que conforman el nivel mínimo de costumbres, creencias y suposiciones que se dan por hecho en cada escuela. Podría parecer un concepto sencillo y con una estructura simple, pero lo cierto es que atañe a las bases, creencias y suposiciones sobre las que se crean y funcionan las instituciones, por lo que abarca un amplio espectro de conceptos e ideas.

\section{Definición de Cultura Escolar}

Atribuir el concepto de cultura a la escuela no es una práctica novedosa (Elías, 2015). Se trata de un término empleado frecuentemente para describir e identificar los procesos de la vida escolar. De forma similar, Waller (2014) define que cada escuela tiene su identidad, sus costumbres y normas, creando un código moral. Encontrar una únıca definición del concepto puede ser complejo debido al gran número de dimensiones a incluir. Por ejemplo, Schein (2018) en un intento de definir el término, elaboró un listado con los factores intervinientes en la cultura escolar, los cuales son los siguientes:

1. Actitudes observables (incluye el idioma y los rituales).

2. Normas que evolucionan en equipos de trabajo.

3. Valores dominantes, sobre todo los establecidos por la organización.

4. Las políticas del centro.

5. Las normas.

6. La filosofía que guía las políticas y las normas.

7. El clima escolar.

Schein (2018) trata de ir un poco más allá y añade un matiz importante a su definición: los componentes de la cultura escolar no aparecen como tales, pues la cultura supone el nivel más profundo de suposiciones y creencias comunes para todos los miembros de una organización, y estas son adquiridas involuntariamente por cada uno de los miembros; son intrínsecas en uno mismo. Sientan las bases para dar por ciertos determinados aspectos de la vida dentro de la institución (e.g., costumbres, normas, maneras de actuar, creencias y suposiciones), además de dotar a la organización una visión tanto de sí misma como de su contexto.

Otros autores como Deal \& Kennedy han tratado de proveer una definición más simple definiendo cultura escolar como "la manera que tenemos aquí de hacer las cosas" (1983, p. 501). Esta nueva definición deja de lado el aspecto intrínseco mencionado por Schein (2018). Muchos autores inciden en el aspecto informal y no escrito de la cultura escolar. Incluso se llega a mencionar que tan solo puede ser aprendida y adquirida cuando se rompe una de las normas no escritas; por tanto, las metáforas, las costumbres, los rituales, los mitos, los símbolos, las historietas, y el sentido del humor también 
conforman la cultura escolar (Stoll \& Fink, 1999).

\section{Tipología de Escuela Según su Cultura}

Considerando las definiciones anteriores, resulta evidente la existencia de tantas culturas como escuelas. Aun así, por muy cierta que sea la afirmación anterior, es ineficiente desde el punto de vista científico. Así pues, en aras de hallar patrones y características comunes, se ha tratado de crear categorías o tipologías de cultura escolar para establecer un orden. Rosenholtz (1989) diferenció la escuela flexible (se siente libre para focalizar toda su atención en las prioridades que tiene la escuela) y la inmovilista (busca esa libertad en las exigencias externas).

Esta definición, sin embargo, puede quedarse incompleta al tratar de agrupar todas las escuelas en dos únicas categorías. Es por ello, que otros autores han tratado de abarcar un espectro más amplio. Este es el caso de Stoll \& Fink (1999) que fueron más allá presentando un modelo de cinco categorías atendiendo solamente a dos variables: la efectividad (basada en los resultados académicos de la escuela) y el grado de mejora (el esfuerzo realizado por una escuela para mejorar). Esta diferenciación se puede observar en la figura 1.

\section{Figura 1}

Tipos de cultura escolar según Stoll \& Fink

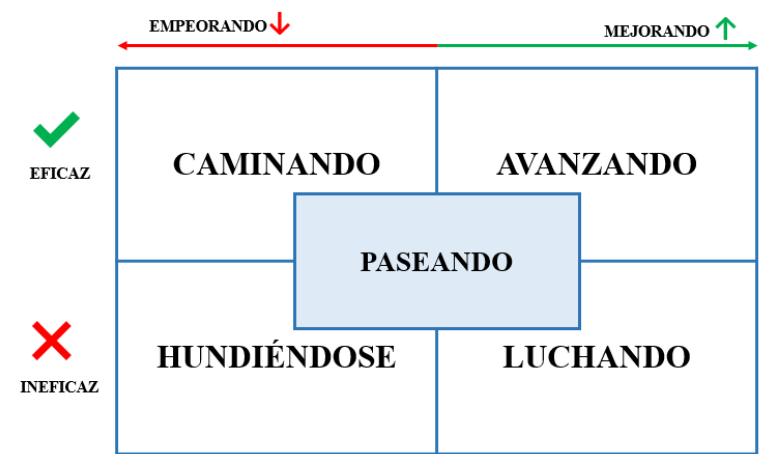

Nota. Stoll \& Fink (1999) proponen este modelo basándose en el estado que tiene la escuela en determinado momento (eficaz o ineficaz) y el esfuerzo que hace para mejorar o mantener esa situación, elaboración propia (2021).

Atendiendo al modelo de las dos variables descritos por Stoll \& Fink (1999), recogidos en la figura 1 , se desarrolla a continuación las características de cada tipo de escuela. Para entender estos modelos, hay que tener en cuenta que la cultura escolar es un proceso dinámico, por lo que, a lo largo del tiempo, ante circunstancias cambiantes de la propia escuela y de su propio entorno, esta podrá pasar por diferentes de las tipologías abajo descritas.

1. Las escuelas que avanzan son eficientes, donde la gente trabaja activamente en grupo para responder a un contexto cambiante.

2. Las escuelas que caminan se perciben como eficientes, logrando buenos resultados con una calidad mejorable. Se conforman con buenos resultados, pero no atienden a las necesidades a largo plazo de su alumnado.

3. Las escuelas que pasean, por su parte, están en medio. Puede parecer que tienen intención de mejorar, pero a paso muy lento. Pueden llegar a proyectar una imagen de una buena escuela, pero necesitan de un estímulo externo para mejorar realmente.

4. Las escuelas que luchan son conscientes de ser poco eficaces, a pesar de efectuar un gran esfuerzo para serlo. Normalmente consiguen sus objetivos debido al nivel de esfuerzo realizado. Es importante que el personal acepte la necesidad de mejorar.

5. Las escuelas que se hunden fracasan y no son efectivas. Los profesores no están preparados (porque no quieren o porque no pueden) para enfrentarse a un proceso de cambio.

\section{Estabilidad de la Cultura, Subculturas y la Escuela como Sistema de Flujos}

Para Stoll \& Fink (1999), quienes proporcionaban este punto de vista sobre los tipos de cultura, creen que la cultura escolar se vuelve estática una vez ha terminado de desarrollarse, partiendo de la idea básica de que la cultura escolar nace junto con la escuela. En contraposición, otros autores 
como Bolman \& Deal (1991) entienden la cultura escolar como un proceso, porque los nuevos miembros la van adquiriendo de quienes ya estaban en la escuela; y, a la vez, como un producto, porque es el resultado del conocimiento de todos los miembros de la escuela).

La cultura escolar, sin embargo, ni como proceso ni como producto, no es una única. Dentro de la cultura predominante en la escuela se crean varias subculturas (ya sea entre alumnos, profesores o familias); de hecho, algunos autores como McLaughlin \& Marsh (1990) y Huberman (1992) describen la cultura predominante en la escuela como la mezcla de todas las subculturas. En ese aspecto, lo más frecuente suele ser la existencia de una cultura que predomina sobre las demás, generalmente impulsada por la dirección del centro; pero, debido a la necesidad de los humanos a relacionarse entre ellos, se crean subculturas (Siskin, 1994).
Conociendo esta realidad de culturas escolares y atendiendo al enfoque de la organización escolar, es preciso entender la escuela como un sistema de flujos. SantosGuerra (1997) recoge que la representación tradicional de la estructura de una organización, como la escuela, es un organigrama; sin embargo, la autora apostilla que, en contraposición a lo que se cree, un organigrama esconde más información de la que ofrece. Se menciona esta idea bajo el pretexto de que, muchas veces, el poder formal no coincide con el poder real. Para comprender el motivo de esta situación, Santos-Guerra (1997) aludió la existencia de personas en lugares y puestos estratégicos, generalmente elegidos por la gente con poco poder, para la toma de decisiones al gusto de quienes los han colocado en dichos puestos. Así, ha de entenderse la escuela, y por ende su cultura, como un sistema de flujos, como se recoge en la tabla 1.

Tabla 1

La organización como un sistema de flujos

\begin{tabular}{cl}
\hline \multicolumn{1}{c}{ TIPO DE FLUJO } & \multicolumn{1}{c}{ DEFINICIÓN } \\
\hline Flujo de trabajo & $\begin{array}{l}\text { Se da en el ámbito de trabajo; tiene varias direcciones: de niveles superiores a inferiores } \\
\text { (explicaciones del profesor), horizontal (trabajo de los alumnos), de niveles inferiores a } \\
\text { superiores (cuando un alumno pide ayuda...). }\end{array}$ \\
\hline Flujo de control & $\begin{array}{l}\text { Se puede relacionar con el control social que ejerce la escuela. El control, generalmente, se } \\
\text { establece mediante la toma de decisiones; estas, tomadas por gente de rango superior, } \\
\text { afectan a los de rango inferior. }\end{array}$ \\
\hline Flujo de información & $\begin{array}{l}\text { Formal, centrada en el trabajo. Tiene muchas direcciones: de arriba a abajo (pedir } \\
\text { orientaciones sobre el trabajo), de abajo a arriba (quejas, pedir permisos), horizontal } \\
\text { (interacciones relacionadas con el trabajo), y con el entorno (consejos dirigidos a la escuela, } \\
\text { peticiones de colaboración...). }\end{array}$ \\
\cline { 2 - 3 } & $\begin{array}{l}\text { Informal, de múltiples direcciones. Las personas de rango más alto, como la dirección del } \\
\text { centro, tratan de evitar este tipo de flujo, pero siempre sucede. Como consecuencia, crea } \\
\text { áreas de poder en la cultura o en las subculturas. La información puede ser centrada en el } \\
\text { trabajo (en ocasiones puede facilitar el funcionamiento de la escuela) o en un ámbito más } \\
\text { social (rumores, cotilleos...). }\end{array}$ \\
\hline
\end{tabular}

Nota. Esta tabla recoge los tres tipos de flujo que se dan en un centro escolar de acuerdo con (Santos-Guerra, 1997). Cuando se distinguen flujos verticales, se alude a distintos grupos según el poder que tienen en la organización (dirección, docentes, alumnado), mientras que la horizontal hace referencia a las personas del mismo grupo, elaboración propia (2021).

Como se ha podido observar, la cultura de la escuela abarca a su vez muchos otros conceptos que a priori podrían no parecer relacionados entre sí, ni con la cultura escolar. Si bien puede parecer un concepto compartimentado, se hace necesario considerar la escuela como una organización para entenderlo bien.

\section{La Problemática de no Atender a la Cultura Escolar}

Como se puede inferir de la definición de la cultura escolar, incluyendo todas sus dimensiones, el término abarca prácticamente todo lo que sucede dentro de la escuela. No obstante, como se menciona, una de las pocas maneras de entender por completo el término $\mathrm{y}$, por tanto, de conocer bien la cultura de un 
centro, es trabajando o estudiando en esa escuela; pero un proceso de estas características requiere una gran cantidad de tiempo.

El requerimiento de tiempo es normal, porque cuando se va a un sitio nuevo, no se sabe muy bien cómo trabajan allí, qué costumbres tienen, por lo que no es inmediato el proceso de adquirir la cultura de una institución... Desde el punto de vista de la investigación educativa, para compensar dicha dificultad, se dispone de otras herramientas de medición de la cultura escolar que no pasan por efectuar una estancia en el centro. Una de estas alternativas es el cuestionario, actualmente ya empleado como instrumento de recogida de datos para medir los niveles de implicación escolar. Por tanto, se estimó coherente y adecuado añadir un segundo apartado a dicho cuestionario, que recoja únicamente datos sobre la cultura escolar.

La problemática surgió cuando han de establecerse las variables que se estudiarán en ese cuestionario, pues pudo parecer laborioso abstraer en unas pocas preguntas la esencia de lo que sucede dentro de la escuela. Hargreaves (1995), por ejemplo, proponía medir el control social que el centro ejerce sobre sus miembros y la cohesión social, estableciendo el modelo descrito a continuación. De tal manera, se logra, simplemente atendiendo a dos variables, describir el ambiente de la escuela:

1. Control social alto y cohesión social alta: escuela invernadero. Claustrofóbica, demasiada presión sobre los participantes.

2. Control social alto y cohesión social baja: escuela tradicional, vigilante $\mathrm{y}$ formal.

3. Control social bajo y cohesión social alta: escuela del bienestar, tranquila y humanitaria.

4. Control social bajo y cohesión social baja: escuela atomizada y solitaria. No hay sentimiento de pertenencia.

En cualquier caso, atender únicamente a dos variables podría resultar insuficiente para ofrecer datos sólidos y fiables. Siguiendo por esa misma línea, para conocer mejor lo que sucede en la escuela, se propone añadir dos variables más orientadas a obtener datos más amplios:

(1) Solidez de la cultura escolar (abarca subculturas, participación de las familias, flujos, presencia $\mathrm{y}$ conocimiento de normas escritas/no escritas... el resto de los componentes y factores presentes en la cultura escolar).

(2) Relación alumno-profesor.

Por tanto, este ensayo presentó un método que consta de siete variables (agrupadas en implicación escolar y cohesión social) que se aplicaría a todas aquellas investigaciones que tuvieran como objetivo estudiar la implicación escolar de un determinado grupo de alumnos. Estas siete variables son:

A. Bloque 1: Implicación escolar:

○ Variable 1: Implicación emocional.

- Variable 2: Implicación conductual.

- Variable 3: Implicación cognitiva.

B. Bloque 2: Cultura escolar:

○ Variable 4: Solidez y arraigo de la cultura escolar.

- Variable 5: Control social.

- Variable 6: Cohesión social.

- Variable 7: Relación alumnoprofesor.

Luego, han de establecerse las variables independientes de la investigación o del estudio que se esté llevando a cabo, como pueden ser la etapa académica de escolarización, la edad, el sexo, entre otras. Estas dependerán del objetivo general del estudio y funcionarán como piezas clave para entender en función de qué y cómo varían las siete variables propuestas.

El objetivo de este novedoso método fue dotar de valor real a los datos recogidos al medir la implicación escolar, ofreciendo, al mismo tiempo, a la comunidad educativa herramientas para establecer relaciones de 
causa-efecto. En la actualidad, se dice que, por ejemplo, "los alumnos de 15 años tienen una implicación conductual baja (participan poco)" y no se aporta ninguna información más, por lo que se están dando a conocer datos carentes de valor real.

En contraposición, si también se investiga la cultura escolar, se dispone de datos y herramientas suficientes para conocer qué influye en los niveles de implicación. Partiendo del ejemplo anterior, si además de observar que los alumnos de 15 años tienen una implicación conductual baja, se advirtió que la cohesión social es baja, o que el alumnado valora negativamente su relación con el cuerpo docente, entonces -y únicamente entonces- se contó con herramientas $y$ datos suficientes para establecer relaciones causa-efecto. Si, por el contrario, se desconoció la cultura escolar, difícilmente se pudo llegar a teorizar sobre el origen o la causa de los niveles de implicación $\mathrm{y}$, en consecuencia, todo intento de tatar de justificar o comprender dichos datos quedó completamente alejado de la realidad escolar donde fueron tomados y basado en teorías.

\section{Conclusión}

La implicación escolar ha resultado ser una gran materia de investigación educativa, no solo por la utilidad de los datos que se recogen, sino porque se ha convertido en un excelente indicador para predecir problemas futuros como el abandono escolar. Además, conviene no olvidar que el objetivo de toda investigación educativa no es solo crear nueva evidencia científica, sino ayudar a toda la comunidad educativa (alunando, docentes, familias...) dotándoles de nuevas herramientas, métodos y datos que puedan ser comprendidos por la población general.

En este aspecto, la investigación de la implicación escolar ha tenido luces $y$ sombras. Ha hecho una excelente labor a la hora de ofrecer datos, estudiar las variables que pueden afectar a la implicación escolar... Pero se ha quedado corta a la hora de brindar un marco a los datos obtenidos. Para ello, ha de entenderse la escuela como una organización, como un ente más, y no solo como un medio para aprender o preparar a los alumnos para el mundo. Como organización, tiene sus normas y costumbres, las cuales indudablemente afectan a la manera en la que el alumno se relaciona con la misma.

Este ensayo, por tanto, propuso ir un paso más allá en la construcción del marco para los datos recogidos de una escuela. Planteó acopiar datos de la cultura escolar para disponer de información útil, como pueden ser los niveles de cohesión y control social, e información del contexto escolar, ofreciendo herramientas para establecer relaciones de causa y efecto. Por ejemplo: en una investigación educativa los datos de los alumnos de un centro indican unos niveles de implicación emocional y conductual bajos. Si no se aporta ningún dato más, dichos datos de los niveles se quedarán cortos, porque, más allá de un valor meramente informativo, no realizan una aportación significante. Además, cualquier intento de encontrar una explicación a esos niveles sería meramente teórica (podría basarse en teorías del desarrollo e investigaciones previas, pero carecería de una visión de donde se han tomado los datos).

En cambio, si se conocieran los datos de la cultura escolar, se podría ver si los niveles de cohesión y control social son altos o bajos, si se valora positivamente la relación de los alumnos con sus docentes o si existe una cultura bien formada y arraigada en la escuela. Son precisamente estos datos, los relacionados con la cultura escolar, los que realmente realizan una aportación significativa de gran valor, tanto para la comunidad científica como para la comunidad escolar en general. Como se mencionaba al principio, el objetivo de toda investigación ha de ser ayudar a la comunidad educativa; en esa misión es preciso conocer también lo que sucede dentro de las escuelas.

Así, se concluye que la comunidad educativa debe disponer de un marco que le permita conocer la realidad de las escuelas. Este marco dotará de información que enriquezca y complemente los datos obtenidos en la medición de la implicación escolar; y dicho marco, precisamente, lo ofrece la investigación sobre la cultura escolar. De esta manera, atendiendo a la 
cultura e implicación escolar, se consigue combinar dos conceptos esenciales muy valiosos en la investigación educativa. Ambos logran ofrecer un punto de vista completo y enriquecido, no empleado hasta el momento. Se aplica así un nuevo enfoque al ámbito de la implicación escolar tomando como eje principal la misma, donde se perciben la escuela y el aprendizaje como un proceso global y multidisciplinar.

\section{Referencias}

Bolman, L. G. \& Deal, T. E. (1991). Leadership and management effectiveness: A multi-frame, multisector analysis. Human resource management, 30(4), 509-534.

Connell, J. P. (1990). Context, self, and action: A motivational analysis of self-system processes across the lifespan. D. Cicchetti (Ed.), The self in transition: Infancy to childhood (pp. 61-97). University of Chicago Press.

Corno, L. \& Mandinach, E. (1983). The role of cognitive engagement in classroom learning and motivation. Educational Psychologist, $18, \quad 88-108$ https://dx.doi.org/10.1080/00461528309529266

Costa, S. \& Tabernero, C. (2012). Rendimiento académico y autoconcepto en estudiantes de educación secundaria obligatoria según el género. Revista Iberoamericana de Psicología y salud, 3(2), 175-193.

Deal, T. E. \& Kennedy, A. A. (1983). Culture: A new look through old lenses. The Journal of Applied Behavioral Science, 19(4), 498-505. https://dx.doi.org/10.1177/002188638301900411

Elías, M. E. (2015). La cultura escolar: Aproximación a un concepto complejo. Revista Electrónica Educare, 19(2), 285-301. http://dx.doi.org/10.15359/ree.192.16

Finn, J. D. (1989). Withdrawing from school. Review of Educational Research, 59, 117-142.

Fredricks, J. A., Blumenfeld, P., Friedel, J. \& Paris, A (2005). School engagement. L. Lipman (Ed.) What do children need to flourish? (pp. 305-321). Springer.

Hargreaves, D. H. (1995). School culture, school effectiveness and school improvement. School Effectiveness and School Improvement, 6(1), 23-46. https://dx.doi.org/10.1080/0924345950060102

Huberman, M. (1992). Teacher development and instructional mastery. A. Hargreaves y M. Fullan (Eds.), Understanding teacher development, (pp. 216-241). Longman Publishers.

McLaughlin, M. W. \& Marsh, D. D. (1990). Staff development and school change. Schools as collaborative cultures: Creating the future now, 213232.

Newman, F.M., Wehlage, G.G. \& Lamborn, S.D. (1992). The significance and sources of student engagement. F. M., Newman (Ed), Student Engagement and Achievement in American Secondary Schools (pp. 11-39). Teachers College Press.

Ramos-Díaz, E., Rodríguez-Fernández, A., Ros, I. \& Antonio-Agirre, I. (2017). Implicación escolar y autoconcepto multidimensional en una muestra de estudiantes de secundaria. Revista Complutense de Educación, 28(4), 1103-1118.

Rosenholtz, S. J. (1989). Teachers' workplace: The social organization of schools. Addison-Wesley Longman Limited.

Santos-Guerra, M. A. (1997). La luz del prisma: para comprender las organizaciones educativas. Aljibe.

Schein, E. H. (2018). Origanizational Culture and Leadership (5. ed.). Wiley.

Siskin, L. S. (1994). Realms of knowledge: Academic departments in secondary schools. Falmer Press.

Stoll, L. \& Fink, D. (1999). El poder de la cultura de la escuela. L. Stoll, eta D. Fink (Eds.), Para cambiar nuestras escuelas: reunir la eficacia y la mejora (pp. 141-169). Octaedro.

Voelkl, K. (1997). Identification with school. American Journal of Education, 105, 294-318

Waller, W. (2014). The Sociology of Teaching. Martino Fine Books. 